\title{
BREASTFEEDING: KNOWLEDGE AND PRACTICES OF MOTHERS
}

\author{
F. BENNAOUI',2,N.EL IDRISSI SLITINE ${ }^{1,2}$, F.M.R MAOULAININE ${ }^{1,2}$ \\ 1-Neonatal Intensive Care Department, Mohammed VI University Hospital and Research \\ 2-Team for Childhood, Health and Development, Marrakech School of Medicine, Cadi Ayyad University, Marrakech, \\ Morocco.
}

\section{Introduction}

Exclusive breastfeeding covers the nutritional needs of the newborn during the first months of life and decreases the risk of infection.

\section{Objective}

The purpose of this survey was to evaluate the breastfeeding knowledge and practices of mothers who have a newborn hospitalized in neonatal intensive care unit.

\section{Materials and methods}

$>$ Prospective and retrospective study. $>60$ mother-newborn couples.

$>$ Neonatal intensive care unit in Mohamed VI University Hospital in Marrakech.

\section{Results}

$>$ The average age was 30 years with extreme ages ranging from 18 to 43 years old.

$>53 \%$ lived in urban areas.

$>$ Women who were illiterate and had a primary level of education accounted for $61 \%$.

$>32 \%$ of women were primiparous.

$>$ Related marriage was at $18 \%$.

$>72 \%$ of women have already breastfed before, of which $46 \%$ were exclusive.

$>$ The pregnancy was followed at $75 \%$ of parturients.

$>$ Caesarean section was performed at $13 \%$.

$>$ The delivery was in the hospital at $69 \%$.

$>$ Only $32 \%$ had been educated about breastfeeding. The husband participated in $40 \%$.

$>$ The main reason for breastfeeding was the good development of the newborn (52\%).

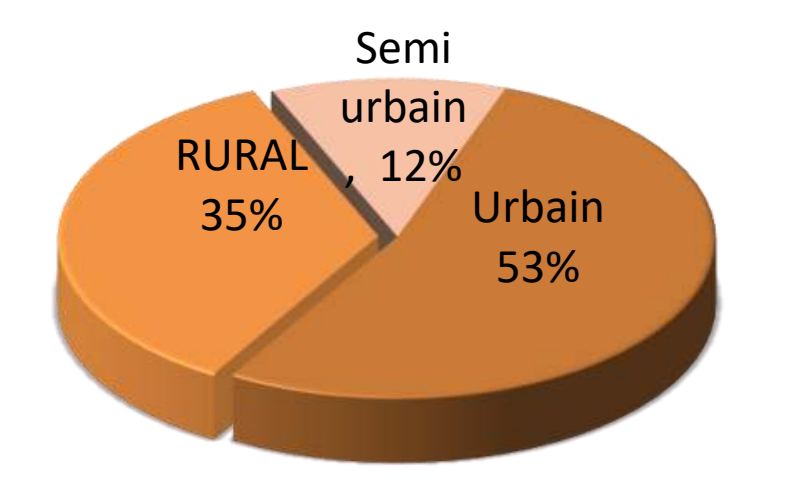

$*$

Before the 1980s, breastfeeding was of little concern to health professionals in Morocco, his practice was universal.

* Towards the end of the 1980s, there was already a decline in breastfeeding in favor of artificial breastfeeding.

Figure 1 : geographic origin Thus, from 1992 to 1997, the exclusive breastfeeding rate increased from $62 \%$ to $46 \%$. The mean duration of breastfeeding also decreased during the same period from 15.5 months to 14 months.

* At present, abandonment of breastfeeding is

a problem public health.

* Indeed, we are currently witnessing a downward trend in this practice in relation to progress in the manufacture, marketing of milk lack of information and awareness among mothers.
Figure 2: Level of sensitization

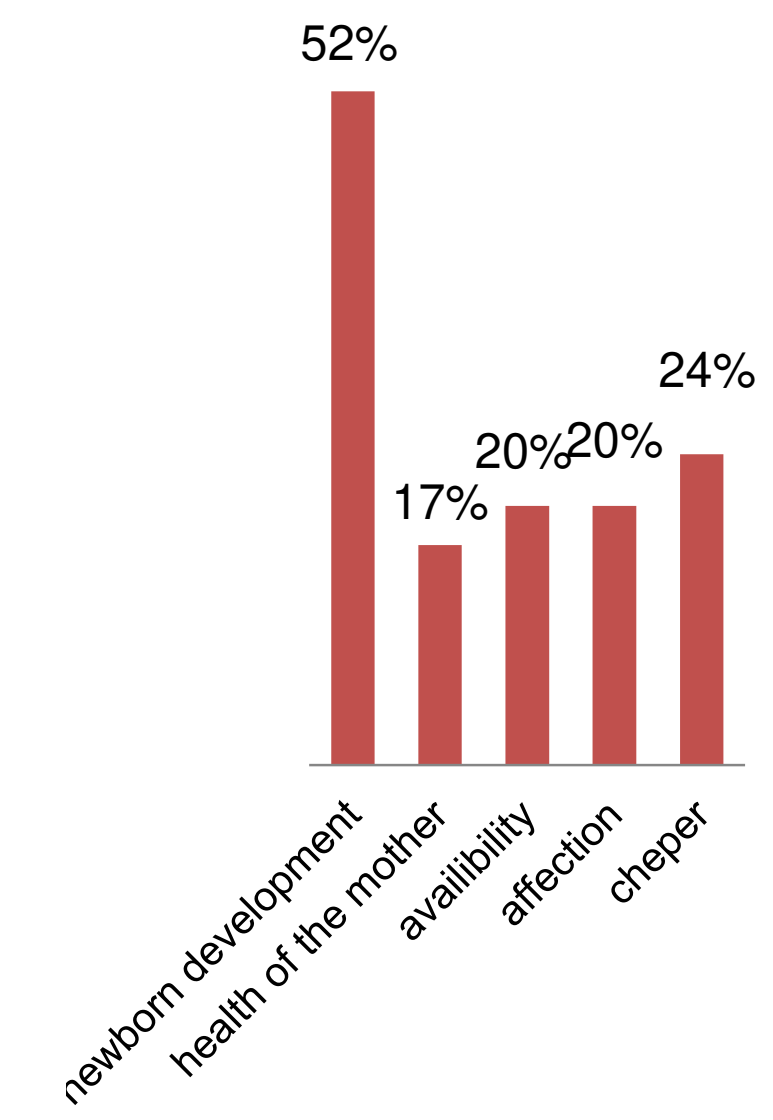

Figure 3: Reasons for breastfeeding.

\section{Conclusion}

The practice of exclusive breastfeeding and partial breastfeeding is marked by wide disparities according to social class, level of education, mode and place of birth. 